\title{
Квантовый транспорт в полупроводниковом нанослое с учетом поверхностного рассеяния носителей заряда
}

\author{
(C) И.А. Кузнецова, О.В. Савенко, Д.Н. Романов
}

Ярославский государственный университет им. П.Г. Демидова, 150003 Ярославль, Россия

E-mail: kuz@uniyar.ac.ru

Поступила в Редакцию 12 апреля 2021 г.

В окончательной редакции 19 апреля 2021 г.

Принята к публикации 19 апреля 2021 г.

Решена задача об электропроводности тонкого проводящего нанослоя с учетом квантовой теории процессов переноса. Толщина слоя может быть сравнима и меньше длины волны де Бройля носителей заряда. Поверхность постоянной энергии имеет вид эллипсоида вращения, главная ось которого параллельна плоскости слоя. Получены аналитические выражения для компонент тензора проводимости, как функции безразмерных толщины, химического потенциала, параметра эллиптичности и параметров шероховатости поверхностей. Выполнен анализ проводимости в предельных случаях вырожденного и невырожденного электронного газа. Проведено сравнение полученных результатов с известными экспериментальными данными для слоя кремния.

Ключевые слова: тонкий слой, уравнение Лиувилля, модель Соффера, электропроводность, изоэнергетическая поверхность.

DOI: 10.21883/FTP.2021.09.51296.26

\section{1. Введение}

В последние десятилетия технологии, связанные с применением тонкопленочных покрытий, активно развиваются. Ведутся исследования по использованию тонких проводящих слоев в микро-, нано- и оптоэлектронике, солнечной энергетике. Современные методы позволяют создавать нанопленки с характерным размером порядка нанометров. Для теоретического изучения электрических характеристик таких нанопленок необходимо учитывать не только поверхностное рассеяние носителей заряда, но и квантование энергетического спектра носителей заряда. Квантовые размерные эффекты могут проявляться по-разному. В металлических пленках могут быть осцилляции зависимостей проводимости от толщины с периодом, равным половине длины волны де Бройля носителя заряда [1-3]. В полупроводниковых пленках наблюдается резкое увеличение сопротивления при малых толщинах [4-6]. Имеется значительное количество теоретических работ, в которых для решения задач об электропроводности тонких пленок и проволок использовался стандартный кинетический метод [7-13]. Однако данный метод неприменим, когда толщина пленки или радиус проволоки сравнимы или меньше длины волны де Бройля носителя заряда.

Для учета квантования энергетического спектра носителей заряда необходимо использовать квантовую теорию явлений переноса. Можно выделить ряд теоретических работ, авторы которых использовали различные методы решения подобных задач. В работах [14-16] использован метод функций Грина, согласно которому потенциал взаимодействия носителей заряда с поверхностью пленки представляет собой малую поправку к собственному гамильтониану системы. Другой способ решения задач заключался в непосредственном расчете интеграла поверхностных столкновений [17-21]. Поверхностное рассеяние рассматривалось как внутризонные и межзонные переходы носителей заряда, рассчитывалась вероятность переходов между состояниями. Однако данные методы решения задач приводят к громоздким математическим расчетам.

В настоящей работе предложен новый метод решения задачи об электропроводности тонкого проводящего слоя с учетом квантовой теории процессов переноса. Согласно рассмотренному методу, поверхностное рассеяние носителей заряда учитывается через граничные условия Соффера [22], полученные в результате решения квантово-механической задачи о взаимодействии электронной волны с поверхностью слоя.

Типичные полупроводниковые материалы, такие, как кремний и германий, имеют поверхность постоянной энергии, состоящую из нескольких эллипсоидов вращения. Поэтому случай произвольного закона дисперсии и эллипсоидальной изоэнергетической поверхности представляет интерес и рассмотрен в настоящей работе.

\section{2. Постановка задачи}

Рассмотрим тонкий проводящий слой толщины $a$, к которому приложено постоянное электрическое поле напряженности $\mathbf{E}$ (рис. 1).

Слой является достаточно тонким, так что его толщина может быть сравнима или меньше длины волны де Бройля носителей заряда. В этом случае необходимо учитывать квантование энергетического спектра 


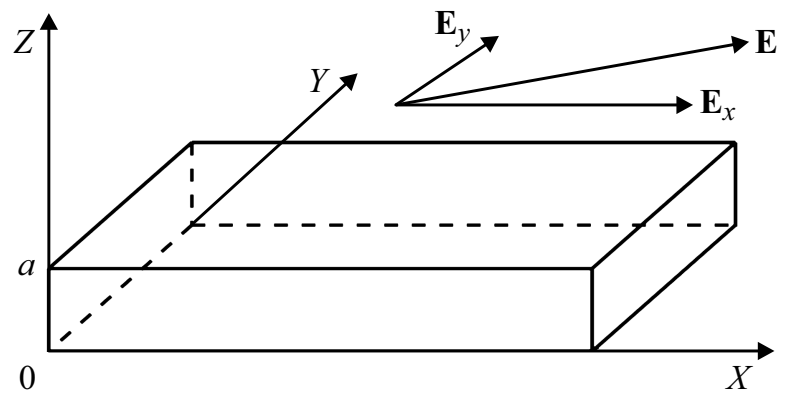

Рис. 1. Тонкий проводящий слой в продольном электрическом поле.

носителей заряда в направлении, перпендикулярном поверхности слоя. Предполагается, что изоэнергетическая поверхность имеет вид эллипсоида вращения, главная ось которого параллельна оси $X$. Тогда выражение для полной энергии электрона (дырки) на $n$-м энергетическом уровне будет иметь вид

$$
\varepsilon_{n}=\frac{m_{\|}}{2} v_{x}^{2}+\frac{m_{\perp}}{2} v_{y}^{2}+\varepsilon_{1} n^{2}
$$

где $m_{\|}$и $m_{\perp}-$ соответственно продольная и поперечная эффективные массы электрона (дырки), $\varepsilon_{1}=(\pi \hbar)^{2} /\left(2 m_{\perp} a^{2}\right)-$ собственное значение энергии носителя заряда на первом энергетическом уровне.

Как отмечалось выше, решение задачи об электропроводности слоя будем проводить в рамках квантовой теории явлений переноса [23]. Система носителей заряда описывается оператором плотности:

$$
\hat{\rho}(t)=\sum_{n} W_{n}\left|\psi_{n}(t)\right\rangle\left\langle\psi_{n}(t)\right|,
$$

который подчиняется уравнению Лиувилля:

$$
i \hbar \frac{\partial \hat{\rho}(t)}{\partial t}=[\hat{H}(t), \hat{\rho}(t)]
$$

Здесь $\psi_{n}(t)$ - волновая функция системы носителей заряда, $W_{n}$ - статистический вес, характеризующий вероятность того, что система носителей находится в состоянии $\psi_{n}, \hat{H}(t)$ - гамильтониан системы.

Будем предполагать, что отклонение системы носителей заряда от состояния равновесия невелико:

$$
\hat{\rho}=\hat{\rho}^{(0)}+\hat{\rho}^{(1)},
$$

где $\hat{\rho}^{(0)}$ - оператор плотности, описывающий равновесное состояние системы, $\hat{\rho}^{(1)}-$ неравновесная поправка.

Далее мы покажем, что при условии (4) уравнение (3) можно привести к виду, аналогичному кинетическому уравнению Больцмана в приближении времени релаксации $\tau$ :

$$
\begin{gathered}
v_{z} \frac{\partial f_{n}^{(1)}}{\partial z}+\frac{e \mathbf{E}}{\hbar} \frac{\partial f_{n}^{(0)}}{\partial \mathbf{k}}=-\frac{f_{n}^{(1)}}{\tau}, \\
f_{n}^{(0)}=\frac{1}{1+\exp \left(\left(\varepsilon_{n}-\mu\right) / k_{0} T\right)} .
\end{gathered}
$$

Здесь $T, \mu, k_{0}-$ соответственно температура, химический потенциал и постоянная Больцмана, $e-$ заряд электрона (дырки), $\hbar-$ постоянная Планка. Отметим, что роль диагональных элементов матрицы плотности в уравнении (5) играет функция распределения носителей заряда $f_{n}$.

Поверхностное рассеяние носителей заряда будем учитывать при помощи граничных условий Соффера, накладываемых на уравнение (5):

$$
\begin{gathered}
\left\{\begin{array}{l}
f_{n}^{(1)+}=q_{1}\left(g_{1}, \theta\right) f_{n}^{(1)-} \quad \text { при } z=0, \\
f_{n}^{(1)-}=q_{2}\left(g_{2}, \theta\right) f_{n}^{(1)+} \quad \text { при } z=a
\end{array}\right. \\
q_{1,2}\left(g_{1,2}, \theta\right)=\exp \left(-\left(4 \pi g_{1,2} \cos \theta\right)^{2}\right), \\
g_{1,2}=g_{s 1,2} / \lambda_{\mathrm{B}}
\end{gathered}
$$

Здесь $f_{n}^{(1) \pm}$ - соответственно функции распределения электронов (дырок) с положительной и отрицательной проекциями волнового вектора на ось $Z, g_{s 1,2}-$ соответственно среднеквадратичная высота поверхностного рельефа нижней и верхней поверхности, $\lambda_{\mathrm{B}}-$ длина волны де Бройля носителей заряда, $\theta-$ угол падения носителя заряда на внутреннюю поверхность слоя.

Найденные функции $f_{n}^{(1) \pm}$ позволят рассчитать такие характеристики, как плотность тока и интегральная проводимость.

\section{3. Вывод кинетического уравнения}

Выведем из уравнения Лиувилля (3) уравнение, аналогичное кинетическому уравнению Больцмана. Если представить гамильтониан $\hat{H}$ как сумму собственного гамильтониана $\hat{H}_{0}$ и зависящего от времени потенциала $\hat{V}(t)$, индуцирующего переходы между собственными состояниями, то уравнение для матричных элементов оператора плотности $\rho_{n^{\prime} n}$ принимает вид [23]

$$
i \hbar \frac{\partial \rho_{n^{\prime} n}}{\partial t}=\left(\varepsilon_{n^{\prime}}-\varepsilon_{n}\right) \rho_{n^{\prime} n}+\sum_{n_{1}}\left(V_{n^{\prime} n_{1}} \rho_{n_{1} n}-\rho_{n^{\prime} n_{1}} V_{n_{1} n}\right)
$$

Здесь $\varepsilon_{n}-$ собственное значение гамильтониана $\hat{H}_{0}$, $V_{n^{\prime} n_{1}}$ - матричный элемент оператора $\hat{V}$.

Используя (10), запишем уравнение для диагональных элементов матрицы плотности $f_{n}=\rho_{n n}$ :

$$
\frac{\partial f_{n}}{\partial t}=-\frac{i}{\hbar} \sum_{n_{1}}\left(V_{n n_{1}} \rho_{n_{1} n}-\rho_{n n_{1}} V_{n_{1} n}\right) .
$$

Левая часть уравнения (11) является аналогом полевого члена кинетического уравнения Больцмана и характеризует изменение функции $f_{n}$ в пространстве координат и волновых векторов в результате действия внешних полей. Правая часть аналогична столкновительному члену, характеризующему изменение функции распределения в результате рассеяния носителей заряда. 
Начнем с рассмотрения левой части уравнения. Анализ полевого члена уравнения (11) аналогичен [24] для квазиклассического случая. Разница состоит в том, что функция $f_{n}$ зависит не от шести аргументов, а от пяти (три компоненты координаты и две компоненты волнового вектора). Однако, поскольку электрическое поле направлено вдоль слоя, изменение функции распределения в пространстве волновых векторов может происходить только в плоскости $\left(k_{x}, k_{y}\right)$. Анализируя левую часть уравнения (11) аналогично [24], запишем

$$
\frac{\partial f_{n}}{\partial t}=v_{z} \frac{\partial f_{n}}{\partial z}+\frac{e \mathbf{E}}{\hbar} \frac{\partial f_{n}}{\partial \mathbf{k}} .
$$

Здесь $v_{z}$ имеет смысл скорости распространения вероятностной волны вдоль оси $Z$. Покажем, что эта скорость принимает лишь дискретный ряд значений. Решением нестационарного уравнения Шредингера для задачи о квантовой яме с бесконечно высокими стенками является функция:

$$
\begin{aligned}
& \psi(z, t)=A \exp \left(-i \varepsilon_{n} t / \hbar\right) \sin \left(q_{n} z\right) \\
& =A \exp \left(-i\left(\frac{\varepsilon_{n} t}{\hbar}+\frac{\pi n z}{a}\right)\right)+A \exp \left(-i\left(\frac{\varepsilon_{n} t}{\hbar}-\frac{\pi n z}{a}\right)\right),
\end{aligned}
$$

которую можно интерпретировать как совокупность бегущих навстречу друг другу плоских волн с волновым числом $q_{n}=\pi n / a$ и одинаковой фазовой скоростью:

$$
v_{z n}=\frac{\pi n \hbar}{m a}=v_{1} n,
$$

где $v_{1}$ - скорость распространения волны в первой подзоне.

Рассмотрим правую часть уравнения (11). Мы будем рассматривать рассеяние на точечных дефектах и примесях, находящихся только в объеме образца, поверхностное рассеяние будем учитывать при помощи граничных условий. Предположим, что носители заряда, находящиеся в состояниях $\psi_{n}$ и $\psi_{n_{1}}$, характеризуются волновыми векторами $\mathbf{k}$ и $\mathbf{k}_{1}$, и рассмотрим рассеяние носителя заряда из состояния $\psi_{n}$ в состояние $\psi_{n_{1}}$. Потенциал рассеяния и его матричный элемент имеют вид [24]:

$$
\begin{gathered}
V(\mathbf{r})=\sum_{i} V_{0}\left(\mathbf{r}-\mathbf{R}_{i}\right), \\
V_{n i}=V_{\mathbf{q}}=\int V(\mathbf{r}) \exp (-i \mathbf{q r}) d^{3} r \\
=V_{\mathbf{q}}^{0} \sum_{i} \exp \left(-i \mathbf{q} \mathbf{R}_{i}\right),
\end{gathered}
$$

где $\mathbf{R}_{i}-$ радиус-вектор $i$-го узла, $\mathbf{q}-$ разность волновых векторов $\mathbf{k}$ и $\mathbf{k}_{1}, V_{0}$ и $V_{\mathbf{q}}^{0}-$ соответственно потенциал рассеяния носителей заряда на одной примеси и его матричный элемент.
Уравнение для диагональных элементов матрицы плотности запишется следующим образом:

$$
\frac{\partial f_{\mathbf{k}}}{\partial t}=-\frac{i}{\hbar} \sum_{\mathbf{q}}\left(V_{\mathbf{q}} \rho_{\mathbf{k}-\mathbf{q}, \mathbf{k}}-V_{-\mathbf{q}} \rho_{\mathbf{k}, \mathbf{k}-\mathbf{q}}\right)
$$

или

$$
\frac{\partial f_{\mathbf{k}}}{\partial t}=-\frac{i}{\hbar} \sum_{\mathbf{q}} V_{\mathbf{q}}\left(\rho_{\mathbf{k}-\mathbf{q}, \mathbf{k}}-\rho_{\mathbf{k}, \mathbf{k}+\mathbf{q}}\right) .
$$

Поскольку потенциал рассеяния зависит только от разности волновых векторов $\mathbf{q}$, удобнее проводить суммирование по q.

Найдем недиагональные элементы матрицы плотности $\rho_{\mathbf{k}-\mathbf{q}, \mathbf{k}}$.Исходя из уравнения (10) запишем уравнения для нахождения $\rho_{\mathbf{k}-\mathbf{q}, \mathbf{k}}$ :

$$
\begin{aligned}
i \hbar \frac{\partial \rho_{\mathbf{k}-\mathbf{q}, \mathbf{k}}}{\partial t}= & \left(\varepsilon_{\mathbf{k}-\mathbf{q}}-\varepsilon_{\mathbf{k}}\right) \rho_{\mathbf{k}-\mathbf{q}, \mathbf{k}} \\
& +\sum_{\mathbf{q}_{1}} V_{\mathbf{q}_{1}}\left(\rho_{\mathbf{k}-\mathbf{q}-\mathbf{q}_{1}, \mathbf{k}}-\rho_{\mathbf{k}-\mathbf{q}, \mathbf{k}+\mathbf{q}_{1}}\right) .
\end{aligned}
$$

В случае малого отклонения системы от состояния равновесия производную функции $f_{\mathbf{k}}$ по времени можно записать как произведение функции на некоторый малый параметр $s$. Учитывая вышесказанное, получим выражение для $\rho_{\mathbf{k}-\mathbf{q}, \mathbf{k}}$ :

$$
\rho_{\mathbf{k}-\mathbf{q}, \mathbf{k}}=\frac{1}{\varepsilon_{\mathbf{k}-\mathbf{q}}-\varepsilon_{\mathbf{k}}+i \hbar s} \sum_{\mathbf{q}_{1}} V_{\mathbf{q}_{1}}\left(\rho_{\mathbf{k}-\mathbf{q}-\mathbf{q}_{1}, \mathbf{k}}-\rho_{\mathbf{k}-\mathbf{q}, \mathbf{k}+\mathbf{q}_{1}}\right) .
$$

Выражение для $\rho_{\mathbf{k}, \mathbf{k}+\mathbf{q}}$ получается из (20) заменой $\mathbf{k} \rightarrow \mathbf{k}+\mathbf{q}:$

$$
\rho_{\mathbf{k}, \mathbf{k}+\mathbf{q}}=\frac{1}{\varepsilon_{\mathbf{k}}-\varepsilon_{\mathbf{k}+\mathbf{q}}+i \hbar s} \sum_{\mathbf{q}_{1}} V_{\mathbf{q}_{1}}\left(\rho_{\mathbf{k}-\mathbf{q}_{1}, \mathbf{k}+\mathbf{q}}-\rho_{\mathbf{k}, \mathbf{k}+\mathbf{q}+\mathbf{q}_{1}}\right) .
$$

Подставляя (20) и (21) в (18), получим выражение

$$
\begin{aligned}
i \hbar \frac{\partial f_{\mathbf{k}}}{\partial t}= & \sum_{\mathbf{q}, \mathbf{q}_{1}} V_{\mathbf{q}} V_{\mathbf{q}_{1}}\left(\frac{\rho_{\mathbf{k}-\mathbf{q}-\mathbf{q}_{1}, \mathbf{k}}-\rho_{\mathbf{k}-\mathbf{q}, \mathbf{k}+\mathbf{q}_{1}}}{\varepsilon_{\mathbf{k}}-\varepsilon_{\mathbf{k}-\mathbf{q}}+i \hbar s}\right. \\
& \left.-\frac{\rho_{\mathbf{k}-\mathbf{q}_{1}, \mathbf{k}+\mathbf{q}_{1}}-\rho_{\mathbf{k}, \mathbf{k}+\mathbf{q}+\mathbf{q}_{1}}}{\varepsilon_{\mathbf{k}}-\varepsilon_{\mathbf{k}-\mathbf{q}}+i \hbar s}\right) .
\end{aligned}
$$

Распишем с учетом (16) произведение $V_{\mathbf{q}} V_{\mathbf{q}_{1}}$ :

$$
\begin{aligned}
V_{\mathbf{q}} V_{\mathbf{q}_{1}}= & V_{\mathbf{q}}^{0} V_{\mathbf{q}_{1}}^{0}\left(\sum_{i \neq j}\left(\exp \left(-i\left(\mathbf{q} \mathbf{R}_{i}+\mathbf{q}_{1} \mathbf{R}_{j}\right)\right)\right)\right. \\
& \left.+\sum_{i}\left(\exp \left(-i\left(\mathbf{q}+\mathbf{q}_{1}\right) \mathbf{R}_{i}\right)\right)\right) .
\end{aligned}
$$

Поскольку точечные дефекты расположены произвольным образом, первая сумма в выражении (23) равна нулю. Отличным от нуля остается второе слагаемое при 
условии, когда сумма векторов $\mathbf{q}$ и $\mathbf{q}_{1}$ равна нулю. С учетом вышесказанного выражение (22) принимает вид

$$
\begin{aligned}
\frac{\partial f_{\mathbf{k}}}{\partial t}= & -\frac{i}{\hbar} \sum_{\mathbf{q}}\left|V_{\mathbf{q}}^{0}\right|^{2} \\
& \times\left(\frac{f_{\mathbf{k}}-f_{\mathbf{k}-\mathbf{q}}}{\varepsilon_{\mathbf{k}}-\varepsilon_{\mathbf{k}-\mathbf{q}}+i \hbar s}+\frac{f_{\mathbf{k}}-f_{\mathbf{k}-\mathbf{q}}}{\varepsilon_{\mathbf{k}-\mathbf{q}}-\varepsilon_{\mathbf{k}}+i \hbar s}\right) .
\end{aligned}
$$

Переходя к пределу $s \rightarrow 0$ и полагая, что

$$
\operatorname{Im}\left(\frac{1}{\varepsilon_{\mathbf{k}}-\varepsilon_{\mathbf{k}-\mathbf{q}}+i 0}\right)=-\pi \delta\left(\varepsilon_{\mathbf{k}}-\varepsilon_{\mathbf{k}-\mathbf{q}}\right),
$$

получим выражение

$$
\begin{aligned}
\frac{\partial f_{\mathbf{k}}}{\partial t} & =-\sum_{\mathbf{k}_{1}} W_{\mathbf{k}_{1} \mathbf{k}}\left(f_{\mathbf{k}}-f_{\mathbf{k}_{1}}\right), \\
W_{\mathbf{k}_{1} \mathbf{k}} & =\frac{2 \pi}{\hbar}\left|V_{\mathbf{q}}^{0}\right|^{2} \delta\left(\varepsilon_{\mathbf{k}}-\varepsilon_{\mathbf{k}_{1}}\right) .
\end{aligned}
$$

Здесь $W_{\mathbf{k}_{1} \mathbf{k}}$ имеет смысл вероятности перехода носителя заряда из состояния $\mathbf{k}$ в состояние $\mathbf{k}_{1}$. В случае упругого рассеяния носителей заряда и малого отклонения системы от состояния равновесия, интеграл столкновений можно выразить через время релаксации [24]:

$$
\sum_{\mathbf{k}_{1}} W_{\mathbf{k}_{1} \mathbf{k}}\left(f_{\mathbf{k}}-f_{\mathbf{k}_{1}}\right)=\frac{f_{\mathbf{k}}^{(1)}}{\tau} .
$$

Учитывая (12), (26) и (28), уравнение на функцию $f_{n}(11)$ будет иметь вид

$$
v_{1} n \frac{\partial f_{n}^{(1)}}{\partial z}+\frac{e \mathbf{E}}{\hbar} \frac{\partial f_{n}^{(0)}}{\partial \mathbf{k}}=-\frac{f_{n}^{(1)}}{\tau} .
$$

Таким образом, уравнение (29) имеет некоторое сходство с кинетическим уравнением Больцмана в квазиклассическом приближении. Поскольку изоэнергетическая поверхность представляет собой эллипсоид вращения, время релаксации будет тензором второго ранга, а функция $f_{n}$ - тензорной функцией:

$$
\tau=\left(\begin{array}{ccc}
\tau_{\|} & 0 & 0 \\
0 & \tau_{\perp} & 0 \\
0 & 0 & \tau_{\perp}
\end{array}\right), f_{n}=\left(\begin{array}{ccc}
f_{n \|} & 0 & 0 \\
0 & f_{n \perp} & 0 \\
0 & 0 & f_{n \perp}
\end{array}\right)
$$

\section{4. Математические расчеты}

Решая уравнение (29) с учетом граничного условия (7), получим выражения для функций распределения носителей заряда $f_{n}^{(1) \pm}$ :

$$
\begin{aligned}
f_{n}^{(1)+}(z) & =-\frac{e\left(v_{x} E_{x}+v_{y} E_{y}\right)}{\tau} \frac{\partial f_{n}^{(0)}}{\partial \varepsilon_{x}}\left(1-\phi_{n}^{+} e^{-\Omega_{n} \xi}\right), \\
f_{n}^{(1)-}(z) & =-\frac{e\left(v_{x} E_{x}+v_{y} E_{y}\right)}{\tau} \frac{\partial f_{n}^{(0)}}{\partial \varepsilon_{y}}\left(1-\phi_{n}^{-} e^{\Omega_{n}(1-\xi)}\right),
\end{aligned}
$$

$$
\begin{gathered}
\phi_{n}^{+}=\frac{\left(1-q_{1}\right)+q_{1}\left(1-q_{2}\right) e^{-\Omega_{n}}}{1-q_{1} q_{2} e^{-2 \Omega_{n}}}, \\
\phi_{n}^{-}=\frac{\left(1-q_{2}\right)+q_{2}\left(1-q_{1}\right) e^{\Omega_{n}}}{1-q_{1} q_{2} e^{2 \Omega_{n}}}, \\
\Omega_{n}=a /\left(v_{1} n \tau\right), \quad \xi=z / a .
\end{gathered}
$$

Полученные функции распределения позволяют рассчитать ток, плотность которого равна

$$
j_{i}=\frac{2 e k_{1}}{(2 \pi)^{3}} \sum_{n} \iint v_{j}\left(\left(f_{n}^{(1)+}\right)_{j i}+\left(f_{n}^{(1)-}\right)_{j i}\right) d k_{x} d k_{y} .
$$

Полагая $v_{1}=\hbar k_{1} / m_{\perp}, \quad v_{x}=\hbar k_{x} / m_{\|}, \quad v_{y}=\hbar k_{y} / m_{\perp}$, распишем выражение (36) для компонент плотности тока на оси $X$ и $Y$ :

$$
\begin{aligned}
& j_{x}=2 e\left(\frac{m_{0}}{h}\right)^{3} v_{1} \sum_{n} \int_{-\infty}^{\infty} \int_{-\infty}^{\infty} v_{x}\left(f_{n \|}^{(1)+}+f_{n \|}^{(1)-}\right) d v_{x} d v_{y}, \\
& j_{y}=2 e\left(\frac{m_{0}}{h}\right)^{3} v_{1} \sum_{n} \int_{-\infty}^{\infty} \int_{-\infty}^{\infty} v_{y}\left(f_{n \perp}^{(1)+}+f_{n \perp}^{(1)-}\right) d v_{x} d v_{y},
\end{aligned}
$$

где введено обозначение $m_{0}=\sqrt[3]{m_{\|} m_{\perp}^{2}}$.

Для вычисления интегралов удобно перейти к системе координат $\left(u_{\|}, \varphi\right)$ в пространстве скоростей. Связь координат $\left(v_{x}, v_{y}\right)$ и $\left(u_{\|}, \varphi\right)$ дается следующей системой уравнений:

$$
\left\{\begin{array}{l}
v_{x}=\sqrt{\frac{2 k_{0} T u_{\|}}{m_{\|}}} \cos \varphi, \\
v_{y}=\sqrt{\frac{2 k_{0} T u_{\|}}{m_{\perp}}} \sin \varphi .
\end{array}\right.
$$

Подставляя (31), (32), (39) в (37) и (38) и проведя необходимые вычисления, получим следующие выражения для проекций плотности тока на оси $X$ и $Y$ :

$$
\begin{aligned}
j_{x}= & \frac{2 e^{2} m_{0}^{2} E_{x} \tau_{\|} \pi \sqrt{u_{1}}}{h^{3}}\left(\frac{2 k_{0} T}{m_{0}}\right)^{3 / 2} \gamma^{2} \sum_{n=1}^{\infty} \ln \left(e^{u_{\mu}-u_{1} n^{2}}+1\right) \\
& \times\left(2+\phi_{n \|}^{+} e^{-\Omega_{n \|} \xi}+\phi_{n \|}^{-} e^{-\Omega_{n \|}(1-\xi)}\right), \\
j_{y}= & \frac{2 e^{2} m_{0}^{2} E_{y} \tau_{\perp} \pi \sqrt{u_{1}}}{h^{3}}\left(\frac{2 k_{0} T}{m_{0}}\right)^{3 / 2} \frac{1}{\gamma} \sum_{n=1}^{\infty} \ln \left(e^{u_{\mu}-u_{1} n^{2}}+1\right) \\
& \times\left(2+\phi_{n \perp}^{+} e^{-\Omega_{n \perp} \xi}+\phi_{n \perp}^{-} e^{-\Omega_{n \perp}(1-\xi)}\right) .
\end{aligned}
$$

Здесь введены следующие обозначения:

$$
u_{\mu}=\frac{\mu}{k_{0} T}, \quad u_{1}=\frac{m_{\perp} v_{1}^{2}}{2 k_{0} T}, \quad \gamma=\frac{m_{\perp}}{m_{0}} .
$$

Локальную проводимость можно рассчитать исходя из локального закона Ома:

$$
j_{i}=\sigma_{i j} E_{j}
$$


Интегральная проводимость определяется как интеграл от локальной проводимости по толщине слоя. Поскольку изоэнергетическая поверхность имеет форму эллипсоида вращения, главная ось которой параллельна оси $X$, проводимость представляет собой тензор второго ранга с нулевыми недиагональными компонентами:

$$
\begin{gathered}
\sigma_{a}=\left(\begin{array}{cc}
\sigma_{a \|} & 0 \\
0 & \sigma_{a \perp}
\end{array}\right), \\
\sigma_{a \|}=\int_{0}^{a} \sigma_{\|} d z=a \int_{0}^{1} \frac{j_{x}}{E_{x}} d \xi, \\
\sigma_{a \perp}=\int_{0}^{a} \sigma_{\perp} d z=a \int_{0}^{1} \frac{j_{y}}{E_{y}} d \xi .
\end{gathered}
$$

Подставляя выражения (40) и (41) в (45) и (46), получим выражения для продольных и поперечных компонент тензора проводимости:

$$
\begin{gathered}
\sigma_{\|, \perp}=\sigma_{0} a \Sigma_{\|, \perp}\left(x_{0}, x_{\lambda}, \gamma, g_{1}, g_{2}, u_{\mu}\right) \\
\sigma_{0}=\frac{n_{v} e^{2} \tau_{0 v}}{m_{0}}, \quad \tau_{0 v}=\sqrt[3]{\tau_{v \|} \tau_{v \perp}^{2}} \\
\Sigma_{\|, \perp}=\frac{\sqrt{u_{0 v}}}{2 x_{0} I_{1 / 2} \Phi_{\|, \perp}(\gamma) \kappa} \sum_{n=1}^{\infty} \ln \left(\exp \left(u_{\mu}-u_{1} n^{2}\right)+1\right) \\
\times\left(1-\chi\left(\frac{2 x_{0}^{2} \kappa \sqrt{\gamma}}{n x_{\lambda} \Phi_{\|, \perp}(\gamma)}\right)\right) \\
\chi(p)=\frac{1}{2 p}\left(1-e^{-p}\right) \frac{2-q_{1}-q_{2}+\left(q_{1}+q_{2}-2 q_{1} q_{2}\right) e^{-p}}{1-q_{1} q_{2} e^{-2 p}} \\
q_{1,2}\left(g_{1,2}, \theta\right)=\exp \left(-\left(2 \pi g_{1,2} n / x_{0}\right)^{2}\right) \\
\Phi_{\|}(\gamma)=\frac{1}{\gamma}, \quad \Phi_{\perp}(\gamma)=\sqrt{\gamma}, \quad \kappa=\frac{\sqrt{u_{0}}}{\sqrt{u_{0 v}}} \\
u_{0}=\frac{5}{3} \frac{I}{P}, \quad \sum_{n=1}^{\infty} \int_{0}^{\infty} \frac{u^{s} d u}{\ln \left(\exp \left(u_{\mu}-u_{1} n^{2}\right)+1\right)} \\
\left.I_{s}=\frac{5}{3} \frac{I_{3 / 2}}{I_{1 / 2}}, \quad u_{\mu}\right)+\frac{u_{0 v}}{4 x_{0}^{2}} \\
\sum_{n=1}^{\infty} \int_{u_{1} n^{2}}^{\infty} \frac{x_{0}}{\exp \left(u-u_{\mu}\right)+1} \\
\int_{\mathrm{B} \perp}^{\infty}
\end{gathered}
$$

Здесь $\sigma_{0}-$ классическая проводимость, $n_{v}$ и $\tau_{0 v}-$ соответственно концентрация носителей заряда и эффективное время релаксации в макроскопическом образце; $\Sigma-$ безразмерная проводимость, зависящая от следующих безразмерных параметров: $x_{0}$ - толщина слоя; $x_{\lambda}$ - длина свободного пробега носителей заряда; $\gamma$ - параметр, характеризующий анизотропию изоэнергетической поверхности (будем называть его параметром эллиптичности), $u_{\mu}$ - химический потенциал, $g_{1,2}$ - параметры шероховатости поверхностей слоя. Безразмерные параметры $x_{0}$ и $x_{\lambda}$ обезразмерены на длину волны де Бройля носителей заряда в направлении, перпендикулярном плоскости слоя $\lambda_{\mathrm{B} \perp}$.

Аналогично решается задача в случае, когда главная ось эллипсоида постоянной энергии параллельна оси $Y$. Продольная компонента тензора проводимости будет равна $\sigma_{\perp}$, а поперечная $-\sigma_{\|}$. Если главная ось будет параллельна оси $Z$, т.е. перпендикулярна плоскости слоя, то продольные и поперечные компоненты тензора проводимости будут равны друг другу. Безразмерная проводимость будет иметь вид

$$
\begin{aligned}
\Sigma_{t}= & \frac{\sqrt{u_{0 v}}}{2 x_{0} I_{1 / 2} \Phi_{\perp}(\gamma) \kappa} \sum_{n=1}^{\infty} \ln \left(\exp \left(u_{\mu}-\frac{u_{0 v} n^{2}}{4 x_{0}^{2}}\right)+1\right) \\
& \times\left(1-\chi\left(\frac{2 x_{0}^{2} \kappa}{n x_{\lambda} \gamma \Phi_{\perp}(\gamma)}\right)\right) .
\end{aligned}
$$

\section{5. Предельные случаи}

Рассмотрим случай вырожденного электронного газа $\left(u_{\mu} \gg 1\right)$, соответствующий высокой концентрации, малой эффективной массе носителей заряда и низкой температуре. Равновесная функция распределения принимает вид ступенчатой аппроксимации:

$$
f_{n}^{(0)}\left(\varepsilon_{n}\right)= \begin{cases}0, & 0<\varepsilon_{n}<\varepsilon_{\mathrm{F}} \\ 1, & \varepsilon_{n}>\varepsilon_{\mathrm{F}}\end{cases}
$$

где $\varepsilon_{\mathrm{F}}$ - энергия Ферми.

В этом случае в выражении (49) можно пренебречь единицей, стоящей под логарифмом, и записать:

$$
\ln \left(\exp \left(u_{\mu}-\frac{u_{0 v} n^{2}}{4 x_{0}^{2}}\right)+1\right) \approx u_{\mu}-\frac{u_{0 v} n^{2}}{4 x_{0}^{2}} .
$$

Безразмерная проводимость принимает вид

$$
\begin{aligned}
& \Sigma_{\|, \perp}= \frac{3}{4 x_{0} \Phi_{\|, \perp}(\gamma)} \sum_{n=1}^{N}\left(1-\frac{n^{2}}{4 x_{0}^{2}}\right) \\
& \times\left(1-\chi\left(\frac{2 x_{0}^{2} \sqrt{\gamma}}{n x_{\lambda} \Phi_{\|, \perp}(\gamma)}\right)\right), \\
& N=\operatorname{int}\left(2 x_{0}\right) .
\end{aligned}
$$

Рассмотрим случай невырожденного электронного газа $\left(u_{\mu} \rightarrow-\infty\right)$, соответствующий низкой концентрации, большой эффективной массе и высокой температуре. Равновесная функция распределения носителей заряда будет иметь вид

$$
f_{n}^{(0)}\left(\varepsilon_{n}\right)=\exp \left(\left(\mu-\varepsilon_{n}\right) / k_{0} T\right) .
$$


Выражение, стоящее в экспоненте в формуле (49), является малым числом. Раскладывая логарифм в ряд Тейлора, получим

$$
\ln \left(\exp \left(u_{\mu}-\frac{u_{0 v} n^{2}}{4 x_{0}^{2}}\right)+1\right) \approx \exp \left(u_{\mu}-\frac{u_{0 v} n^{2}}{4 x_{0}^{2}}\right)+\ldots
$$

Безразмерная проводимость принимает вид

$$
\begin{aligned}
\Sigma_{\|, \perp}= & \frac{1}{x_{0} \Phi_{\|, \perp}(\gamma)} \sqrt{\frac{5}{2 \pi}} \sum_{n=1}^{\infty} \exp \left(-\frac{5 n^{2}}{8 x_{0}^{2}}\right) \\
& \times\left(1-\chi\left(\frac{2 x_{0}^{2} \sqrt{\gamma}}{n x_{\lambda} \Phi_{\|, \perp}(\gamma)}\right)\right) .
\end{aligned}
$$

В случае сферически-симметричной энергетической зоны $(\gamma=1)$ продольные и поперечные компоненты тензора проводимости будут равны друг другу:

$$
\begin{aligned}
\Sigma= & \frac{\sqrt{u_{0 v}}}{2 x_{0} I_{1 / 2} \kappa} \sum_{n=1}^{\infty} \ln \left(\exp \left(u_{\mu}-\frac{u_{0 v} n^{2}}{4 x_{0}^{2}}\right)+1\right) \\
& \times\left(1-\chi\left(\frac{2 x_{0}^{2} \kappa}{n x_{\lambda}}\right)\right) .
\end{aligned}
$$

Рассмотрим предельный переход в квазиклассический случай $\left(x_{0} \gg 1, x_{0} / x_{\lambda} \simeq 1\right)$. В этом случае от суммирования по номеру подзоны $n$ можно перейти к интегрированию. В результате получим следующее выражение:

$$
\begin{aligned}
\Sigma_{\|, \perp}= & 1-\frac{1}{2 I_{1 / 2} x_{0} \sqrt{u_{0}}} \int_{0}^{\infty} \int_{0}^{\pi / 2} \sin ^{3} \theta \cos \theta \frac{u^{2} \exp \left(u-u_{\mu}\right)}{\left(\exp \left(u-u_{\mu}\right)+1\right)^{2}} \\
& \times \chi\left(\frac{\sqrt{u_{0}} x_{0} \sqrt{\gamma}}{\sqrt{u} \cos \theta \Phi_{\|, \perp}(\gamma)}\right) d u d \theta .
\end{aligned}
$$

В выражении (65) под $x_{0}$ понимается отношение толщины слоя к длине свободного пробега носителей заряда.

\section{6. Обсуждение результатов}

На рис. 2 и 3 изображены зависимости безразмерной продольной компоненты тензора интегральной проводимости от безразмерной толщины $x_{0}$ при значении параметра эллиптичности $\gamma$, равном 1 (рис. 2) и 3 (рис. 3). Наблюдается общая тенденция в уменьшении проводимости с уменьшением толщины как вырожденного, так и невырожденного электронного газа, что связано с уменьшением числа разрешенных энергетических состояний носителей заряда. В случае вырожденного электронного газа зависимость проводимости от толщины ведет себя осциллирующим образом с одинаковым периодом осцилляций, равным $\lambda_{\mathrm{B}} / 2$. В случае невырожденного газа осцилляций не наблюдается. Заметим, что относительное различие между зависимостями проводимости от толщины, рассчитанными для

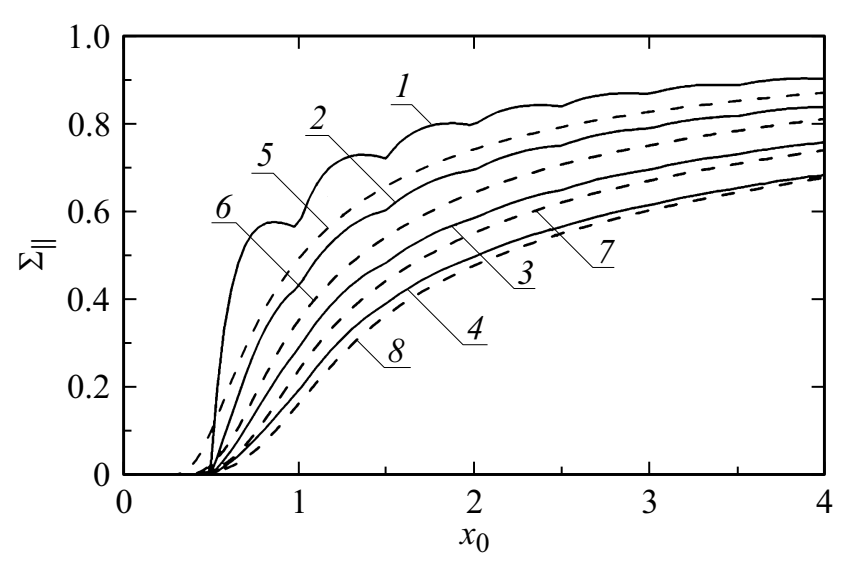

Рис. 2. Зависимости безразмерной продольной компоненты тензора интегральной проводимости $\Sigma_{\|}$от безразмерной толщины $x_{0}$ при значениях $x_{\lambda}=10, \gamma=1: 1,5-$ $g_{1}=g_{2}=0 ; 2,6-g_{1}=g_{2}=0.04 ; 3,7-g_{1}=g_{2}=0.07$; $4,8-g_{1}=g_{2}=0.1$. Сплошные кривые $1-4$ построены для случая вырожденного электронного газа, пунктирные 5-8для случая невырожденного электронного газа.

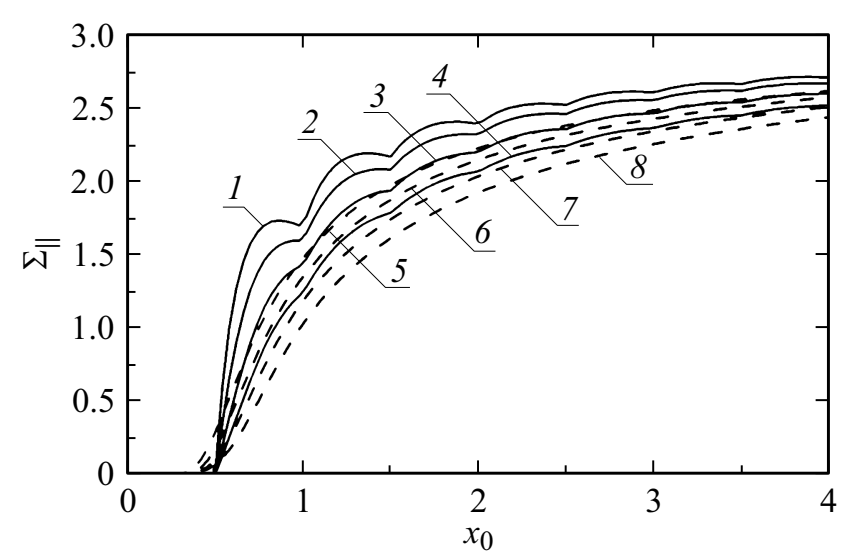

Рис. 3. Зависимости безразмерной продольной компоненты тензора интегральной проводимости $\Sigma_{\|}$от безразмерной толщины $x_{0}$ при значениях $x_{\lambda}=10, \gamma=3: 1,5$ $g_{1}=g_{2}=0 ; 2,6-g_{1}=g_{2}=0.04 ; 3,7-g_{1}=g_{2}=0.07$; $4,8-g_{1}=g_{2}=0.1$. Сплошные кривые $1-4$ построены для случая вырожденного электронного газа, пунктирные $5-8-$ для случая невырожденного электронного газа.

случаев вырожденного и невырожденного газа, имеет максимумы при значениях $x_{0}=(2 n+1) \lambda_{\mathrm{B}} / 4$ и минимумы при значениях $x_{0}=n \lambda_{\mathrm{B}} / 2$ (где $n-$ положительное целое число). С увеличением толщины и параметров шероховатости осцилляции зависимостей проводимости от толщины становятся менее ярко выраженными, а при значениях $x_{0}>3$ и $g_{1}, g_{2}>0.03$ осцилляции практически не наблюдаются. На рис. 2 и 3 наблюдается интересная закономерность: с увеличением параметра эллиптичности относительное различие между кривыми 1-4 и 5-8 уменьшается, т.е. чем больше параметр эллиптичности, тем меньшее влияние на проводимость оказывает поверхность слоя. Данный эффект можно объ- 


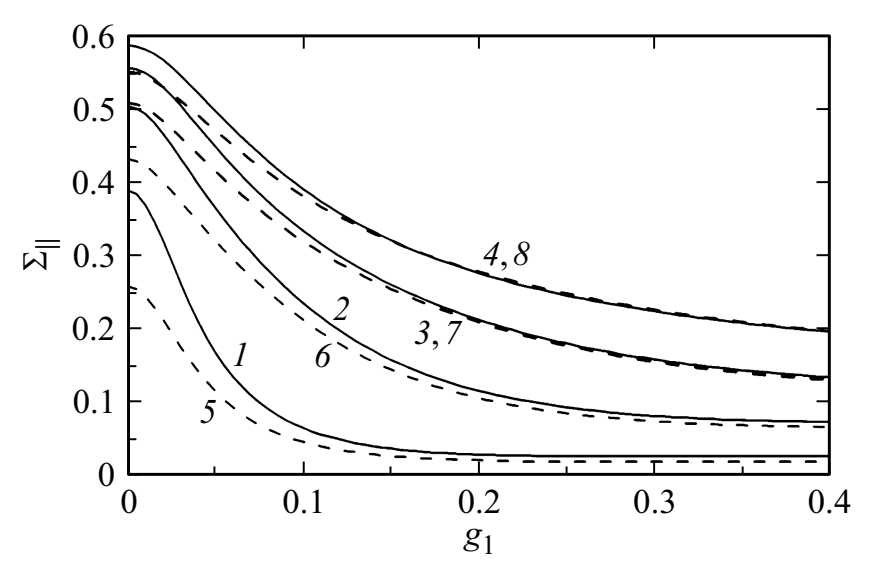

Рис. 4. Зависимости безразмерной продольной компоненты тензора интегральной проводимости $\Sigma_{\|}$от параметра шероховатости нижней поверхности $g_{1}$ при значениях $x_{\lambda}=10$, $k=0.7, g_{2}=0: 1,5-x_{0}=0.75 ; 2,6-x_{0}=1.25 ; 3,7-$ $x_{0}=1.75 ; 4,8-x_{0}=2.25$. Сплошные кривые построены для случая вырожденного электронного газа, пунктирные - для случая невырожденного электронного газа.

яснить следующим образом. С увеличением $\gamma$ поперечная эффективная масса становится больше, следовательно, поперечная скорость носителя заряда будет меньше продольной. Это обстоятельство приводит к уменьшению поверхностных столкновений носителей заряда и, как следствие, к уменьшению влияния поверхностного рассеяния.

На рис. 4 построены зависимости безразмерной продольной компоненты тензора интегральной проводимости от параметра шероховатости нижней поверхности $g_{1}$. Выбор значений безразмерной толщины, при которых строились зависимости $\Sigma_{\|}\left(g_{1}\right)$, был основан на том, чтобы при нулевом значении параметра шероховатости различие между расчетами, полученными для случаев вырожденного и невырожденного газа, было максимальным (см. подп. к рис. 2 и 3). На рисунке видно, что с увеличением шероховатости проводимость уменьшается, что связано с увеличением влияния поверхностного рассеяния носителей заряда на проводимость. Наиболее резкое уменьшение проводимости с ростом шероховатости наблюдается при значениях $0<g_{1}<0.2$, а при $g_{1}>0.4$ проводимость практически не изменяется. Отметим, что с увеличением шероховатости относительное различие между сплошными и пунктирными кривыми уменьшается, а различие между кривыми, отвечающими разным значениям $x_{0}$, возрастает.

На рис. 5 изображены зависимости безразмерной продольной и поперечной компонент тензора интегральной проводимости от параметра эллиптичности $\gamma$. На рисунке видим, что с увеличением $\gamma$ продольная проводимость возрастает, а поперечная уменьшается. Это можно объяснить увеличением поперечной эффективной массы носителей заряда с увеличением $\gamma$ и, как следствие, с уменьшением скорости носителя вдоль оси $Y$. При значении $\gamma=1$, соответствующем сферически-симметричной энергетической зоне, продольная и поперечная проводимости совпадают друг с другом.

На рис. 6 построены зависимости безразмерной продольной компоненты тензора интегральной проводимости от безразмерного химического потенциала $u_{\mu}$. Сплошные кривые построены при нулевом значении шероховатости поверхности, а пунктирные кривые - при значении $g_{1}=g_{2}=0.02$. При отрицательных значениях химического потенциала проводимость не изменяется. В диапазоне $0<u_{\mu}<5$ проводимость возрастает с увеличением $u_{\mu}$. При значениях $u_{\mu}$ больше 5 возникает следующая ситуация. Если толщина слоя равна $n \lambda_{\mathrm{B}} / 2$, где $n$ - целое число (кривые 2 и 4 ), проводимость

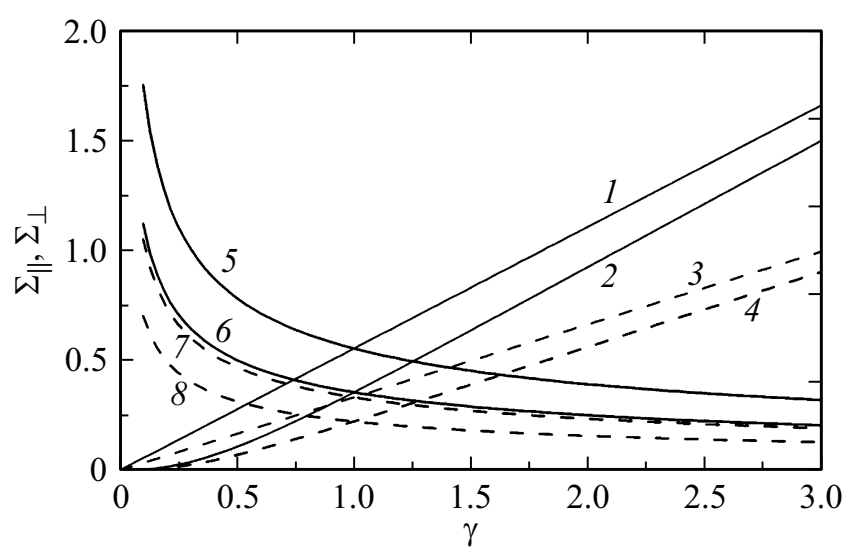

Рис. 5. Зависимости безразмерной продольной $\Sigma_{\|}$(кривые $1-4)$ и поперечной $\Sigma_{\perp}$ (кривые $5-8$ ) компонент тензора интегральной проводимости от параметра эллиптичности $\gamma$ при значениях $x_{\lambda}=10, x_{0}=0.75: 1,3,5,7-g_{1}=g_{2}=0$; $2,4,6,8-g_{1}=g_{2}=0.03$. Сплошные кривые построены для случая вырожденного электронного газа, пунктирные - для случая невырожденного электронного газа.

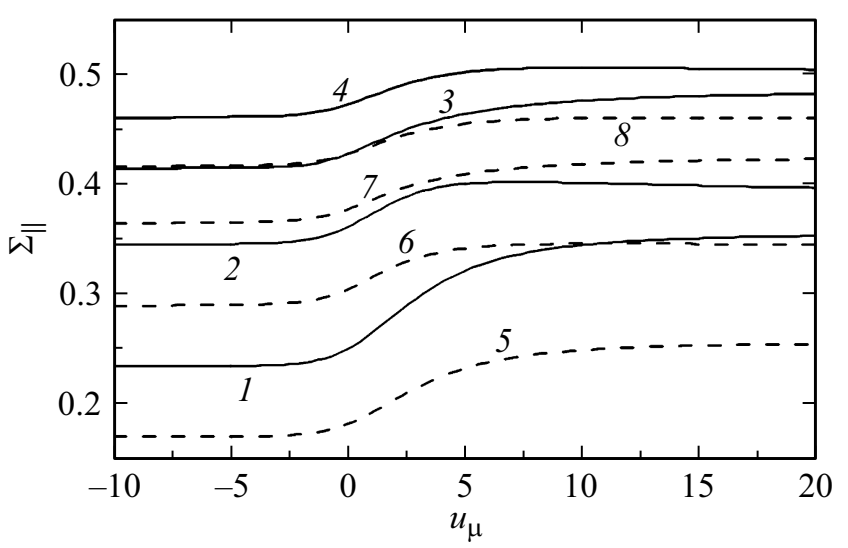

Рис. 6. Зависимости безразмерной продольной $\Sigma_{\|}$компоненты тензора интегральной проводимости от безразмерного химического потенциала $u_{\mu}$ при значениях $x_{\lambda}=10, \gamma=0.7$, $g_{1}=g_{2}=0$ (сплошные кривые), $g_{1}=g_{2}=0.02$ (пунктирные кривые): $1,5-x_{0}=0.75 ; 2,6-x_{0}=1 ; 3,7-x_{0}=1.25$; $4,8-x_{0}=1.5$. 


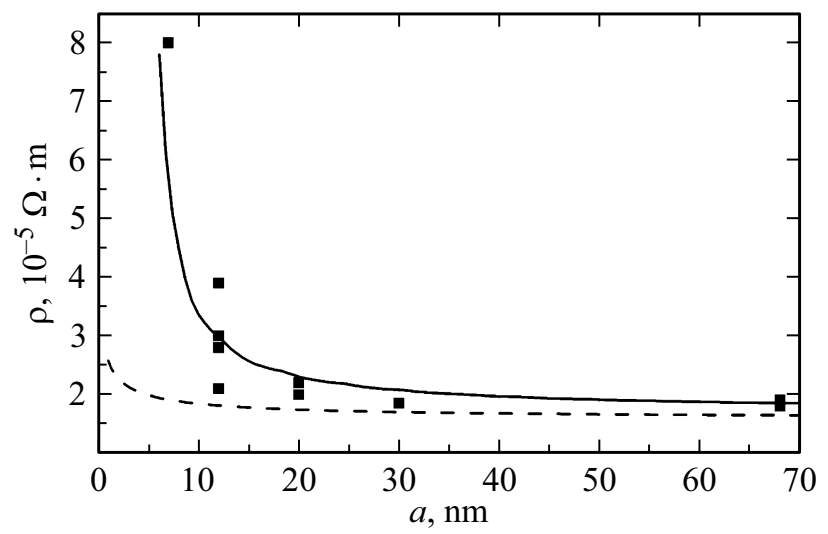

Рис. 7. Зависимости удельного сопротивления нанопленки кремния от толщины. Точки - экспериментальные данные работы [6]. Сплошная кривая - теоретический расчет при значениях $g_{1}=g_{2}=0.05, \gamma=0.6, \Lambda=50 \mathrm{Hм}, \lambda_{\mathrm{B}}=12$ нм, пунктирная кривая - теоретический расчет в квазиклассическом приближении $\left(a \gg \lambda_{\mathrm{B}}\right)$.

практически не изменяется с изменением $u_{\mu}$, а в других случаях (кривые 1 и 3 ) проводимость продолжает плавно возрастать с увеличением $u_{\mu}$.

На рис. 7 построены зависимости удельного сопротивления тонкой пленки кремния, выращенной на подложке $\mathrm{SiO}_{2}$. Сплошная кривая - теоретический расчет с учетом квантования энергетического спектра носителей заряда, пунктирная кривая - теоретический расчет без учета квантования (квазиклассический случай). Точки экспериментальные данные работы [6]. Образцы кремния легированы мышьяком с помощью металлоорганической газофазной эпитаксии. Концентрация примеси составляет $\sim 10^{20} \mathrm{~cm}^{-3}$, т. е. электронный газ можно считать вырожденным. Поскольку изоэнергетическая поверхность кремния представляет собой систему, состоящую из шести симметрично расположенных эллипсоидов вращения, проводимость равна сумме составляющих проводимостей от каждого эллипсоида:

$$
\sigma=2 \sigma_{0}\left(\Sigma_{\|}+\Sigma_{\perp}+\Sigma_{t}\right)
$$

где $\Sigma_{\|}$и $\Sigma_{\perp}$ определяются по формуле (49), а $\Sigma_{t}-$ по формуле (56).

Теоретические расчеты согласуются с экспериментальными данными при значениях параметров шероховатости 0.05. Заметим, что экспериментальная зависимость сопротивления от толщины показывает резкое увеличение удельного сопротивления при толщинах, сравнимых или меньших 10 нм. Из графиков видно, что пунктирная кривая, соответствующая квазиклассической теории, согласуется с экспериментом только при толщинах > 20 нм и не показывает скачка удельного сопротивления. Сплошная кривая, рассчитанная в рамках рассмотренной квантовой модели процессов переноса, хорошо описывает резкое увеличение сопротивления. Возможное объяснение роста удельного сопротивления при малых толщинах заключается в уменьшении количества разрешенных энергетических уровней, на которые могут попадать носители заряда из валентной зоны в зону проводимости. В результате этого концентрация носителей заряда уменышается, а удельное сопротивление возрастает.

\section{7. Заключение}

В настоящей работе построена теоретическая модель электропроводности тонкого проводящего нанослоя с учетом квантования энергетического спектра носителей заряда. Показано, что в случае вырожденного электронного газа зависимости проводимости от толщины ведут себя осциллирующим образом, период осцилляций составляет половину длины волны де Бройля носителей заряда. С увеличением шероховатости поверхностей и толщины слоя осцилляции затухают. В случае невырожденного газа осцилляции зависимостей проводимости от толщины не наблюдаются. Максимальное относительное различие между расчетами, полученными для случаев вырожденного и невырожденного электронного газа, наблюдается при зеркальном рассеянии носителей заряда и значении безразмерной толщины, равном 0.75 .

Сравнительный анализ теоретических расчетов с экспериментальными данными для пленки кремния показывает адекватность построенной теоретической модели, описывающей резкое увеличение удельного сопротивления при толщинах, сравнимых или меньших 10 нм. Полученные результаты могут быть использованы для решения таких задач, как высокочастотная электропроводность, электропроводность 2D канала с треугольной формой потенциальной ямы и т.д.

\section{Финансирование работы}

Исследование выполнено при финансовой поддержке РФФИ в рамках научного проекта № 19-32-90008.

\section{Конфликт интересов}

Авторы заявляют, что у них нет конфликта интересов.

\section{Список литературы}

[1] E. Condreaa, A. Nicorici. Solid State Commun., 150, 118 (2010).

[2] Е.В. Демидов, В.М. Грабов, В.А. Комаров, А.Н. Крушельницкий, А.В. Суслов, М.В. Суслов. ФТП, 53 (6), 736 (2019).

[3] K.M. Tsysar, E.M. Smelova, A.M. Saletsky, V.G. Andreev. Thin Sol. Films, 710, 138263 (2020).

[4] В.Г. Голубев, Л.Е. Морозова, А.Б. Певцов, Н.А. Феоктистов. ФТП, 33 (1), 75 (1999).

[5] I. Miccoli, F. Edler, H. Pfnur, S. Appelfeller, M. Dahne, K. Holtgrewe, S. Sanna, W.G. Schmidt, C. Tegenkamp. Phys. Rev. B, 93, 125412 (2016). 
[6] J. MacHale, F. Meaney, N. Kennedy, L. Eaton, G. Mirabelli, M. White, K. Thomas, E. Pelucchi, D.H. Petersen, R. Lin, N. Petkov, J. Connolly, C. Hatem, F. Gity, L. Ansari, B. Long, R. Duffy. J, Appl. Phys., 125, 225709 (2019).

[7] K. Moors, B. Sorée, W. Magnus. J. Appl. Phys., 118 (12), 124307 (2015).

[8] L. Moraga, K.F. Arenas, R. Henriquez, S. Bravo, B. Solis. Physica B: Condens. Matter, 499, 17 (2016).

[9] M. César, D. Gall, H. Guo. Phys. Rev. Appl., 5, 054018 (2016).

[10] M. De Clercq, K. Moors, K. Sankaran, G. Pourtois, Sh. Dutta, Ch. Adelmann, W. Magnus, B. Sorée. Phys. Rev. Mater., 2, 033801 (2018).

[11] I.A. Kuznetsova, D.N. Romanov, A.A. Yushkanov. Phys. Scripta, 94, 115805 (2019).

[12] П.А. Кузнецов, О.В. Савенко, А.А. Юшканов. ЖТФ, 90 (12), 2002 (2020).

[13] A.A. Yushkanov, O.V. Savenko, I.A. Kuznetsova. Phys. Scripta, 95 (4), 045805 (2020).

[14] L. Sheng, D.Y. Xing, Z.D. Wang. Phys. Rev. B, 51, 7325 (1995).

[15] D. Ketenoglu, B. Ünal. Physica A: Statistical Mech. and its Appl., 391, 3828 (2012).

[16] R.C. Munoz, C. Arenas. Appl. Phys. Rev., 4, 011102 (2017).

[17] A.E. Meyerovich, A. Stepaniants. J. Phys.: Condens. Matter, 12, 5575 (2000).

[18] A.E. Meyerovich, I.V. Ponomarev. Phys. Rev. B, 65, 155413 (2002).

[19] B. Feldman, R. Deng, S.T. Dunham. J. Appl. Phys., 103, 113715 (2008).

[20] S. Chatterjee, A.E. Meyerovich. Phys. Rev. B, 84, 165432 (2011).

[21] R.I. Bihun, Z.V. Stasyuk, O.A. Balitskii. Physica B: Condens. Matter, 487, 73 (2019).

[22] S.B. Soffer. J, Appl. Phys., 38 (4), 1710 (1967).

[23] К. Блум. Теория матрицы плотности и ее приложения (М., Мир, 1983).

[24] А.И. Ансельм. Введение в теорию полупроводников (М., Наука, 1978).

Редактор Г.А. Оганесян

\title{
Quantum transport in a semiconductor nanolayer taking into account surface charge carrier scattering
}

\author{
I.A. Kuznetsova, O.V. Savenko, D.N. Romanov \\ Demidov Yaroslavl State University, \\ 150003 Yaroslavl, Russia
}

Abstract The problem of the conductivity of a thin conductive nanolayer is solved taking into account the quantum theory of transport processes. The layer thickness can be comparable to or less than the de Broglie wavelength of charge carriers. The constant-energy surface has the form of an ellipsoid of revolution with the main axis parallel to the layer plane. Analytical expressions are obtained for the conductivity tensor components as a function of dimensionless thickness, chemical potential, ellipticity parameter, and surface roughness parameters. The conductivity analysis for the limiting cases of a degenerate and non-degenerate electron gas are conducted. The results are compared with known experimental data for a silicon layer. 